\title{
Positive pre-resection pleural lavage cytology is associated with increased risk of lung cancer recurrence in patients undergoing surgical resection: a meta-analysis of 4450 patients
}

\author{
Srdjan Saso, ${ }^{1}$ Christopher Rao, ${ }^{2}$ Hutan Ashrafian, ${ }^{2}$ Sadaf Ghaem-Maghami, ${ }^{1}$ \\ Ara Darzi, ${ }^{2}$ Thanos Athanasiou ${ }^{2}$
}

- Additional appendices are published online only. To view these files please visit the journal online (http://thorax.bmj. com/content/67/6.toc).

${ }^{1}$ Division of Surgery and Cancer, Institute of Reproductive \& Developmental Biology, Imperial College London, Hammersmith Hospital Campus, London, UK ${ }^{2}$ Department of Biosurgery, Surgical Technology and Surgical Epidemiology Unit, Imperial College London, St Mary's Hospital, London, UK

\section{Correspondence to} Srdjan Saso, Clinical Research Fellow, Division of Surgery and Cancer, Institute of Reproductive \& Developmental Biology, Imperial College London, Hammersmith Hospital Campus, Du Cane Road, London W12 0NN, UK:

srdjan.saso@imperial.ac.uk

Received 5 May 2011 Accepted 20 December 2011 Published Online First 27 January 2012

\begin{abstract}
Introduction The value of pleural lavage cytology (PLC) in assessing the prognosis of early stage lung cancer is still controversial. No systematic review has investigated the relationship between PLC and lung cancer recurrence. Our primary goal was to investigate the association between positive pre-resection PLC and pleural, distant and overall tumour recurrence in patients undergoing surgical resection.

Methods Medline, EMBASE and Google Scholar databases were searched up to 2011. All studies reporting relevant outcomes in both patient groups were included. Data were extracted for the following outcomes of interest: overall, local and distant recurrence; and freedom from death (survival-overall and patients with stage I disease only). Random effects meta-analysis was used to aggregate the data.

Sensitivity and heterogeneity analysis were performed.

Results A meta-analysis of eight studies at maximum follow-up demonstrated a significant association between positive pre-resection PLC and increased risk of post-resection overall recurrence $(\mathrm{OR} 4.82,95 \% \mathrm{Cl} 2.45$ to 9.51), pleural recurrence (OR $9.89,95 \% \mathrm{Cl} 5.95$ to 16.44 ) and distant cancer recurrence (OR $3.18,95 \% \mathrm{Cl}$ 1.57 to 6.46). Furthermore, a meta-analysis of 17 studies suggested that positive pre-resection PLC was also associated with unfavourable survival (HR 2.08, 95\% Cl 1.71 to 2.52). These findings were supported by sensitivity analysis.
\end{abstract}

Discussion Positive pre-resection PLC is associated with higher overall, distant and local tumour recurrence and unfavourable patient survival outcomes. This technique may therefore act as a predictor of tumour recurrence and adverse survival. Furthermore, its role in including adjuvant chemotherapy to the management protocol should be investigated within randomised controlled trials.

\section{INTRODUCTION}

Lung cancer is the leading cause of cancer-related mortality throughout the world, accounting for $28 \%$ of all US cancer-related deaths. ${ }^{1}$ The 5 -year overall survival rate remains dramatically low, varying from $8 \%$ in Europe to $15.7 \%$ in the USA. ${ }^{2}$ The most likely explanation lies in the tendency of lung cancer to spread at a microscopic level in its early stage. ${ }^{3}$

\section{Key messages}

What is the key question?

- Can positive pre-resection pleural lavage cytology (PLC) act as a predictor for overall, pleural and distant lung cancer recurrence?

What is the bottom line?

- Positive pre-resection PLC is associated with higher overall, distant and local tumour recurrence.

\section{Why read on?}

- To assess whether positive PLC can 'map out' recurrence risk at an earlier time point and with greater precision than current staging methods, thus altering management strategies.

A need has arisen for methods with the potential to detect microscopic spread. Such methods would allow one to 'map out' recurrence risk at an earlier time point and with greater precision than current staging techniques. ${ }^{4}$ Current staging is based on macroscopic assessment of local, lymphatic and haematogenous spread of cancer. Based on the stage, a decision is made as to whether surgical resection is appropriate or not. ${ }^{5}$ The current TNM staging system does not accurately predict prognosis of recurrence for patients with early stage disease, with a significant number of recurrences and poor survival outcomes occurring in this group. ${ }^{3}$

Pleural lavage cytology (PLC) is a technique which may increase the accuracy of current prediction models for cancer recurrence in early stage disease. ${ }^{6}$ Since its first use in 1958, ${ }^{7}$ several studies have clearly demonstrated a link between the presence of malignant cells in PLC and subsequent early stage pleural dissemination and poor prognosis. $^{8-12}$ However, PLC has not currently been shown to be an independent predictor for recurrence or survival, nor is it included in the present TNM classification.

Two meta-analytical studies have addressed the issue of whether PLC can act as an independent predictor for survival in patients with lung cancer. ${ }^{613}$ Both concluded that positive PLC is a strong 
prognostic factor for survival. However, these meta-analyses did not assess the relationship between positive PLC and recurrence. Additionally, their pre-resection data were derived from only nine trials and 11 centres, respectively, excluding important studies. Finally, the study by Lim et al did not differentiate between pre-resection and post-resection PLC. ${ }^{13}$

The main aim of this study was to test the following three hypotheses:

1. Positive pre-resection PLC is associated with overall lung cancer recurrence.

2. Positive pre-resection PLC is associated with specifically pleural and distant lung cancer recurrence.

3. Positive pre-resection PLC is associated with poor survival outcomes in all patients with lung cancer and in those with stage I disease only.

\section{METHODS}

\section{Literature search}

A literature search was performed using Pubmed, comprising more than 21 million citations for biomedical literature from Medline, life science journals, online books, and Cochrane Library and Google Scholar engines for all relevant studies up until January 2011. The following headings were used in all three databases: Lung cancer, Pulmonary cancer, Lung resection; Pulmonary resection. A Pubmed search was also performed under the following MeSH headings: Lung neoplasm; Non-small cell lung cancer. The results for each heading were subsequently combined with the results from a separate search under the heading: 'Pleural lavage'. The search was expanded using the 'related articles' function and by considering reference lists of all studies, including review articles.

\section{Outcomes of interest}

Positive and negative pre-resection PLC were compared. The primary outcome of interest was whether positive pre-resection PLC was a valid predictor of postoperative pleural and distant cancer recurrence. The secondary outcome of interest was whether positive pre-resection PLC was a valid predictor of worse overall survival in patients with all stages of disease (I-IV), and in patients with stage I disease in particular.

\section{Inclusion and exclusion criteria}

Studies which compared patient outcomes related to positive and negative pre-resection PLC were considered for inclusion. To be included in the actual meta-analysis, the study had to either report recurrence data or results allowing calculation of survival data. If several articles reported on the same patient cohort, the most recent article or the article with the greatest deal of information was selected. No language restrictions were applied.
Studies reporting post-resection PLC were not of interest. Review articles and studies in which it was not possible to extract the primary outcome of interest were excluded.

\section{Data extraction}

Two reviewers (SS and CR) independently performed literature searches and extracted data according to a predefined search protocol. In the case of discrepancy, a consensus decision was achieved by discussion, and if necessary, adjudication by a third reviewer (TA). Patient and cancer characteristics (online appendix A), local and distant cancer recurrence rates (table 1), and survival outcomes were extracted. Pre-resection PLC was defined in all studies as PLC performed immediately after thoracotomy and prior to any pleural parenchymal manipulation. As the authors of those studies described only a single pre-resection PLC in the relevant study methods section, we believe that one pre-resection PLC was performed before it was considered to be negative.

\section{Statistical analysis}

A meta-analysis was conducted according to PRISMA, MOOSE and Cochrane Collaboration guidelines. ${ }^{14-16}$ OR was used as the summary statistic for recurrence rates. An OR $>1$ implied a higher probability of recurrence for the positive PLC group. HR was used as the summary statistic for survival data. Several methods have been described to extract survival data from observational studies, each of these with different limitations. ${ }^{17}$ A HR $>1$ implied a worse survival outcome for the positive PLC group. $\mathrm{OR}$ and $\mathrm{HR}$ were considered statistically significant at the $\mathrm{p}<0.05$ level if the $95 \% \mathrm{CI}$ did not include the value one.

Aggregation of the overall rates of the primary and secondary outcomes were performed with the Mantel-Haenszel method. ${ }^{18}$ We used a random-effects model which assumes that there is variation among studies because this model better accounts for heterogeneity between studies. ${ }^{19} 20$

Analysis was conducted using the statistical software Review Manager V.5.2 (The Cochrane Collaboration, Oxford). For the meta-regression analysis, we used weighted least squares regression with SPSS V.19 for Windows.

\section{Assessment of data validity and heterogeneity (recurrence)}

Three different strategies using quantitative and graphical comparisons were used to assess recurrence data validity and heterogeneity:

1. Sensitivity analysis through examination of the following subgroups:

a. study size (patient number $>350$ )

b. positive PLC percentage ( $<5 \%, 5-10 \%, 10-20 \%,>20 \%)$

c. study quality

d. survival data only: annual follow-up ( $1-5$ years).

Table 1 Cancer recurrence data

\begin{tabular}{|c|c|c|c|c|c|c|c|c|c|c|c|c|c|c|c|c|}
\hline & \multicolumn{2}{|c|}{$\begin{array}{l}\text { Taniguchi } \\
(2009)^{9}\end{array}$} & \multicolumn{2}{|c|}{$\begin{array}{l}\text { Shintani } \\
(2009)^{10}\end{array}$} & \multicolumn{2}{|c|}{$\begin{array}{l}\text { Kawachi } \\
(2009)^{11}\end{array}$} & \multicolumn{2}{|c|}{$\begin{array}{l}\text { Higashiyama } \\
(2009)^{12}\end{array}$} & \multicolumn{2}{|c|}{$\begin{array}{l}\text { Satoh } \\
(2007)^{3}\end{array}$} & \multicolumn{2}{|c|}{$\begin{array}{l}\text { Higashiyama } \\
(1997)^{24}\end{array}$} & \multicolumn{2}{|c|}{$\begin{array}{l}\text { Buhr } \\
(1997)^{25}\end{array}$} & \multicolumn{2}{|c|}{$\begin{array}{l}\text { Okumura } \\
(1991)^{34}\end{array}$} \\
\hline Total & 14 & 282 & 67 & 1182 & 41 & 527 & 89 & 590 & 41 & 812 & 38 & 268 & 132 & 210 & 22 & 135 \\
\hline Pleural recurrence & NS & NS & 3 & 7 & 7 & 31 & 21 & 14 & 8 & 10 & 7 & 5 & 22 & 4 & 2 & 0 \\
\hline Distant metastases & 4 & 27 & 24 & 324 & 13 & 69 & 32 & NS & 23 & 134 & 11 & 70 & 78 & 23 & 9 & 34 \\
\hline Loco-regional recurrence & 2 & 21 & 5 & 53 & 10 & 50 & NS & NS & 2 & 39 & NS & NS & NS & NS & 4 & 13 \\
\hline
\end{tabular}

Neg, negative; NS, not specified; Pos, positive. 
2. Heterogeneity assessment through the $\mathrm{I}^{2}$ statistic: heterogeneity of treatment effects among studies was assessed using the $\mathrm{I}^{2}$ statistic. This represents the proportion of total variation observed among the trials attributable to differences among trials rather than sampling error or chance. ${ }^{21}$

3. Assessment of the following confounders in a metaregression analysis of recurrence data to identify if the results are affected:

a. study size (patient number $>350$ )

b. cut-off of positive PLC (percentage $<5 \%, 5-10 \%, 10-20 \%$, $>20 \%$ )

c. study quality

d. publication year (pre-2005 and post-2005)

4. Graphical exploration to evaluate publication bias in studies reporting recurrence data by assessing for asymmetry and evidence of outliers. ${ }^{22} 23$

\section{Quality scoring}

There was considerable variation among studies in the proportion of patients with a positive PLC. To explore the possible causes of this variation, we created a 'checklist' (online appendix B) which acted as a tool for the assessment of methodological quality and heterogeneity in study design. We attributed one point or a zero to each question answered 'yes' or 'no/not specified', respectively (online appendix C). The median quality score was 11, and studies scoring more than this were deemed to be of high quality and included in the subgroup analysis.

\section{RESULTS}

\section{Selected studies}

The literature search identified 818 studies published between 1991 and 2010. On the basis of title and abstract, 154 articles were obtained and reviewed in full. Eight studies including 4450 patients reported data relevant to the primary outcome of interest: recurrence. ${ }^{3} 9-1224-26$ Data relevant to the secondary outcome of interest-survival-were reported in the same eight studies and nine new studies. ${ }^{8}{ }^{26-33}$ In total, 17 articles met the inclusion and exclusion criteria (figure 1). ${ }^{3} \quad 8-12 \quad 24-34$
Figure 1 Summary of the results of the literature search.

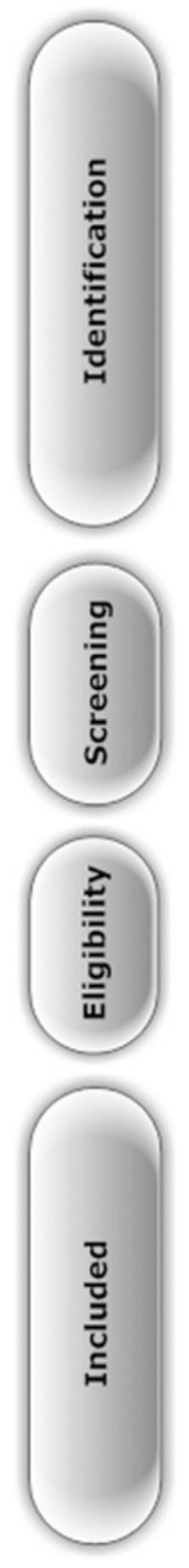

97 records identified through initial search 
Four were prospective observational ${ }^{3} \quad 25 \quad 30 \quad 31$ and 13 were retrospective $e^{8-12} 24 \quad 262829$; all were non-randomised. They contained a total of 9935 patients, of whom 709 (7.14\%) had positive pre-resection PLC.

\section{Meta-analysis of recurrence data}

\section{Overall recurrence}

Positive pre-resection PLC was associated with a higher likelihood of overall, pleural and distant recurrence in comparison to negative pre-resection PLC. Seven studies reported overall recurrence data (figure 2) 3 9-11 242534 for 3771 patients of whom $355(9.41 \%)$ had positive pre-resection PLC. A meta-analysis demonstrated a statistically significant OR of 4.82 (95\% CI 2.45 to $9.51 ; \chi^{2}$ of heterogeneity $\left.40.44(p<0.00001)\right)$.

Pleural recurrence only

Seven studies reported pleural recurrence (4154 patients of whom 430 (10.4\%) had positive pre-resection PLC). OR was statistically significant at $9.89\left(95 \%\right.$ CI 5.95 to $16.44 ; \chi^{2}$ of heterogeneity $8.91(p=0.180)){ }^{3} \quad 10-12242534$

\section{Distant recurrence only}

Seven studies reported distant recurrence (3771 patients of whom 355 (9.41\%) had positive pre-resection PLC). OR was
Figure 2 Forest plot comparing recurrence rates: 'positive PLC versus negative PLC' groups. PLC, pleural lavage cytology.
Positive Negative OR OR

Events Total Events Total Weight M.H, Random, $95 \% \mathrm{Cl} \quad$ M-H, Random, $95 \% \mathrm{Cl}$

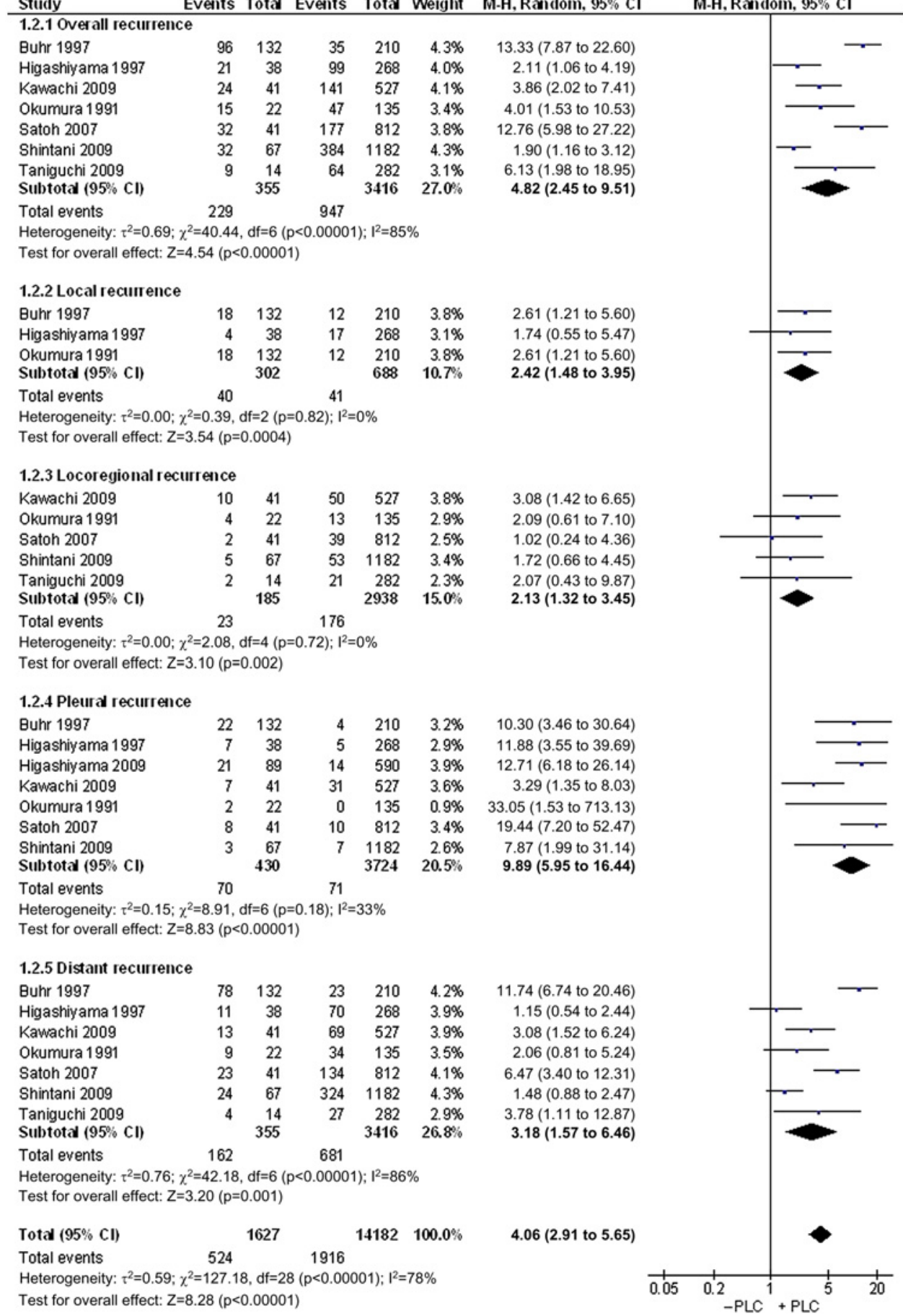


$3.18\left(95 \%\right.$ CI 1.57 to $6.46 ; \chi^{2}$ of heterogeneity 42.25 $(\mathrm{p}<0.00001)) .^{3} 9-11242534$

\section{Meta-analysis of maximum follow-up survival data}

Survival: pathological stage I-IV

In 11 of the 17 studies, $811-1324252728313234$ the association between positive pre-resection PLC and unfavourable survival outcome (all stages) in comparison to negative pre-resection PLC was statistically significant. A meta-analysis of all 17 studies (706 patients (7.14\%) had positive pre-resection PLC) at maximum follow-up supported this finding, with a statistically significant pooled HR of 2.08 (95\% CI 1.71 to $2.52 ; \chi^{2}$ of heterogeneity $54.63(\mathrm{p}<0.00001)$ ) (figure 3).

\section{Survival: pathological stage I}

Nine studies reported survival data for patients with stage I only. ${ }^{3} \quad 12 \quad 24-27 \quad 3132$ Analysis suggested that the association between positive pre-resection PLC and a poor survival outcome was even more pronounced in this group. HR was of statistical significance at 4.20 (random-effects model; 95\% CI 2.65 to 6.65 ; $\chi^{2}$ of heterogeneity $\left.17.90(p<0.02)\right)$.

\section{Sensitivity analysis (overall recurrence)} Study size ( $>350$ patients)

Of the seven studies reporting overall recurrence, three followed up more than 350 patients. ${ }^{3} 1011$ The OR of these three studies (149 patients (5.58\%) had positive pre-resection PLC) was of statistical significance at 4.40 (95\% CI 1.52 to 12.73$)$.

\section{Positive PLC percentage}

Of the seven studies reporting overall recurrence, PLC was positive for $<5 \%$ of the total participants in two studies (55 patients, $4.79 \%){ }^{3}{ }^{9}$ The OR was of statistical significance at 10.01 (95\% CI 5.09 to 19.69). Similarly, PLC was positive for $5-10 \%$ of the total participants also in two studies (108 patients, 5.94\%): OR was of statistical significance at 2.62 (95\% CI 1.31 to 5.24). ${ }^{10} 11$ Finally, two studies (60 patients, $13.0 \%)^{29} 32$ reported $10-20 \%$ of the total participants had positive PLC with similar results. The OR was of statistical significance at 2.65 (95\% CI 1.45 to 4.85$)$.

\section{Quality assessment}

Of the seven studies reporting recurrence, four studies 3102425 were of high quality ( $>11$ matched factors) and were analysed separately. Two hundred and seventy-eight patients (10.1\%) had positive pre-resection PLC. The OR was 5.07 (95\% CI 1.70 to 15.19).

The results of the meta-regression are summarised in table 2 . A sensitivity analysis of the survival data is shown in online appendix D, with an overall results summary presented in table 3 .

\section{Publication bias}

Figure 4 is a funnel plot including all studies (overall recurrence) and reveals four studies 3102425 lying outside the 95\% CI. After exclusion of these outliers, the recurrence outcomes for all patients (OR 4.25; 95\% CI 2.61 to 6.92) and hence our conclusions, were not altered significantly.

\section{DISCUSSION}

Previous reports have highlighted the prognostic importance of PLC with regards to survival. However, there has been no qualitative assessment of the relationship between positive PLC and lung cancer recurrence. A meta-analysis of the eight studies reporting recurrence rates identified an association between positive pre-resection PLC findings and overall, pleural and distant recurrence (statistically significant ORs of 4.82 (95\% CI 2.45 to 9.51$), 9.89$ (95\% CI 5.95 to 16.44 ) and 3.18 (95\% CI 1.56 to 6.46$)$, respectively). This pattern was further demonstrated when analysing local and loco-regional recurrence rates. As predicted, positive pre-resection PLC was also strongly associated with poor survival outcomes with an HR of 2.08 (95\% CI 1.71 to 2.52 ). With respect to survival outcomes, patients with stage I disease are of particular importance because the mainstay of their treatment is surgical resection. On analysis of studies which reported survival data for patients with stage I disease only, the association between the presence of positive PLC and unfavourable survival outcomes was even more pronounced (HR 4.20; $95 \%$ CI 2.65 to 6.65 ).

The results of this meta-analysis allow us to generate a clinical hypothesis: PLC may be a significant adverse prognostic predictor for recurrence (and survival) in patients with (early stage) lung cancer awaiting operative lung resection who may benefit from additional systemic treatment. Our meta-analysis focused on pre-resection PLC only because we wished to test its potential as an additional investigational tool. The point is that by performing PLC prior to surgery, clinicians can use the information to create a more accurate and detailed management
Figure 3 Forest plot comparing survival outcomes: 'positive PLC versus negative PLC' (all patients). PLC, pleural lavage cytology.

\begin{tabular}{|c|c|c|c|c|c|c|c|c|}
\hline Study & $\log$ (Hazard Ratio) & \multicolumn{3}{|c|}{ Positive Negative } & \multirow[t]{2}{*}{ Weight } & \multirow[t]{2}{*}{$\begin{array}{c}\text { HR } \\
\text { IV, Random, } 95 \% \mathrm{Cl}\end{array}$} & \multicolumn{2}{|c|}{$\begin{array}{l}\text { HR } \\
\text { IV, Random, } 95 \% \mathrm{Cl}\end{array}$} \\
\hline \multicolumn{6}{|c|}{ 1.1.1 Survival - maximum follow-up } & & & \\
\hline Aokage 2010 & 0.85 & 0.16 & 65 & 2113 & $8.1 \%$ & 2.34 (1.71 to 3.20$)$ & & $\longrightarrow$ \\
\hline Buhr 1997 & 0.84 & 0.13 & 132 & 210 & $8.7 \%$ & 2.32 (1.80 to 2.99$)$ & & $=$ \\
\hline Dresler 1999 & 0.73 & 0.28 & 17 & 113 & $5.6 \%$ & $2.08(1.20$ to 3.59$)$ & & \\
\hline Higashiyama 1997 & 0.29 & 0.26 & 38 & 268 & $6.0 \%$ & $1.34(0.80$ to 2.22$)$ & & \\
\hline Higashiyama 2009 & 0.51 & 0.16 & 89 & 590 & $8.1 \%$ & 1.67 (1.22 to 2.28$)$ & & $\sim$ \\
\hline Hillerdal 1998 & 0.65 & 0.42 & 17 & 121 & $3.6 \%$ & $1.92(0.84$ to 4.36$)$ & & \\
\hline Kawachi 2009 & 1.09 & 0.08 & 41 & 527 & $9.6 \%$ & 2.97 (2.54 to 3.48 ) & & $=$ \\
\hline Kondo 1993 & 1.2 & 0.16 & 42 & 425 & $8.1 \%$ & 3.32 (2.43 to 4.54$)$ & & 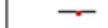 \\
\hline Lim 2004 & 1.17 & 0.27 & 13 & 279 & $5.8 \%$ & 3.22 (1.90 to 5.47$)$ & & \\
\hline Nakagawa 2007 & 1.23 & 0.51 & 27 & 977 & $2.8 \%$ & $3.42(1.26$ to 9.30$)$ & & \\
\hline Okada 2003 & 0.49 & 0.23 & 45 & 955 & $6.6 \%$ & 1.63 (1.04 to 2.56 ) & & \\
\hline Okumura 1991 & 0.89 & 0.32 & 23 & 135 & $4.9 \%$ & 2.44 (1.30 to 4.56$)$ & & \\
\hline Satoh 2007 & 0.22 & 0.22 & 41 & 812 & $6.8 \%$ & $1.25(0.81$ to 1.92$)$ & & \\
\hline Shintani 2009 & 0.07 & 0.18 & 67 & 1182 & $7.6 \%$ & 1.07 (0.75 to 1.53$)$ & & \\
\hline Taniguchi 2009 & 0.69 & 0.61 & 14 & 282 & $2.1 \%$ & $1.99(0.60$ to 6.59$)$ & & \\
\hline Tomita 2005 & 0.84 & 0.59 & 16 & 134 & $2.2 \%$ & 2.32 (0.73 to 7.36$)$ & & \\
\hline Vicidomini 2005 & 0.96 & 0.43 & 19 & $\begin{array}{r}65 \\
9188\end{array}$ & $3.5 \%$ & 2.61 (1.12 to 6.07$)$ & & \\
\hline Subtota $(95 \% \mathrm{Cl})$ & & & 706 & 9188 & $100.0 \%$ & 2.08 (1.71 to 2.52$)$ & & 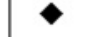 \\
\hline \multicolumn{9}{|c|}{$\begin{array}{l}\text { Heterogeneity: } \tau^{2}=0.10 ; \chi^{2}=54.63, d f=16(p<0.00001) ; l^{2}=71 \% \\
\text { Test for overall effect: } Z=7.37(p<0.00001)\end{array}$} \\
\hline Total $(95 \% \mathrm{Cl})$ & & & 706 & 9188 & $100.0 \%$ & 2.08 (1.71 to 2.52$)$ & & \\
\hline \multicolumn{7}{|c|}{$\begin{array}{l}\text { Heterogeneity: } \tau^{2}=0.10 ; \chi^{2}=54.63, d f=16(p<0.00001) ; l^{2}=71 \% \\
\text { Test for overall effect: } Z=7.37(p<0.00001)\end{array}$} & $\begin{array}{ccc}1 & 1 & 1 \\
0.1 & 0.2 & 0.5\end{array}$ & $\frac{1}{2}$ \\
\hline
\end{tabular}


Table 2 Meta-regression analysis of recurrence data: weighted least squares model

\begin{tabular}{|c|c|c|}
\hline Moderators & Coefficient (95\% CI) & p Value \\
\hline \multicolumn{3}{|l|}{ Overall recurrence } \\
\hline Study quality & $0.05(-0.13$ to 0.24$)$ & 0.49 \\
\hline Publication year & $-0.01(-0.08$ to 0.50$)$ & 0.63 \\
\hline Number of patients & $-0.00(-0.001$ to 0.001$)$ & 0.43 \\
\hline Gender (male) & $-0.00(-0.003$ to 0.001$)$ & 0.16 \\
\hline$+\mathrm{PLC}<5 \%$ & $0.42(-0.42$ to 1.27$)$ & 0.25 \\
\hline$+\mathrm{PLC} 5-10 \%$ & $-0.47(-1.12$ to 0.18$)$ & 0.12 \\
\hline$+\mathrm{PLC} 10-20 \%$ & $-0.34(-1.28$ to 0.60$)$ & 0.40 \\
\hline$+\mathrm{PLC}>20 \%$ & $0.54(-0.25$ to 1.32$)$ & 0.14 \\
\hline \multicolumn{3}{|l|}{ Pleural recurrence } \\
\hline Study quality & $0.11(0.03$ to 0.20$)$ & 0.02 \\
\hline Publication year & $-0.01(-0.07$ to 0.04$)$ & 0.55 \\
\hline Number of patients & $0.00(-0.001$ to 0.001$)$ & 0.84 \\
\hline Gender (male) & $-0.19(-1.43$ to 1.05$)$ & 0.71 \\
\hline$+\mathrm{PLC}<5 \%$ & $0.35(-0.36$ to 1.07$)$ & 0.26 \\
\hline$+\mathrm{PLC} 5-10 \%$ & $-0.51(-0.84$ to 0.17$)$ & 0.01 \\
\hline$+\mathrm{PLC} 10-20 \%$ & $0.21(-0.35$ to 0.77$)$ & 0.38 \\
\hline$+\mathrm{PLC}>20 \%$ & $0.03(-0.87$ to 0.92$)$ & 0.95 \\
\hline \multicolumn{3}{|l|}{ Distant recurrence } \\
\hline Study quality & $0.05(-0.15$ to 0.24$)$ & 0.56 \\
\hline Publication year & $-0.01(-0.07$ to 0.05$)$ & 0.67 \\
\hline Number of patients & $0.00(-0.001$ to 0.001$)$ & 0.44 \\
\hline Gender (male) & $0.75(-0.46$ to 1.95$)$ & 0.17 \\
\hline$+\mathrm{PLC}<5 \%$ & $0.22(-0.57$ to 1.00$)$ & 0.51 \\
\hline+ PLC $5-10 \%$ & $-0.39(-1.11$ to 0.33$)$ & 0.22 \\
\hline+ PLC $10-20 \%$ & $-0.45(-1.40$ to 0.49$)$ & 0.27 \\
\hline$+\mathrm{PLC}>20 \%$ & $0.64(-0.06$ to 1.34$)$ & 0.07 \\
\hline
\end{tabular}

PLC, pleural lavage cytology.

plan than would be possible if PLC was not done. This is because this information will have an impact on whether (or not) to administer chemotherapy and subsequent surgical indications.

Although it is not possible to determine if the relationship between lung cancer cell detection and disease recurrence is causal or simply associative, the detection of lung cancer cells can reflect the presence of extrapleural spread at the time of surgery that might have been missed when using conventional techniques. A significant proportion of patients with stage I cancers and without macroscopic pleural involvement had

Table 3 Summary of the performed meta-analyses (random-effects model)

\begin{tabular}{|c|c|c|c|}
\hline Meta-analysis & HR/OR (95\% CI) & $I^{2}(\%)$ & $\begin{array}{l}\text { Degree of } \\
\text { heterogeneity }\end{array}$ \\
\hline \multicolumn{4}{|l|}{ Overall analysis (recurrence) } \\
\hline Recurrence (overall) & OR 4.82 (2.45 to 9.51$)$ & 85 & High \\
\hline Recurrence (local) & OR 2.42 (1.48 to 3.95 ) & 0 & Low \\
\hline Recurrence (loco-regional) & OR 2.13 (1.32 to 3.45$)$ & 0 & Low \\
\hline Recurrence (pleural) & OR 9.89 (5.95 to 16.44$)$ & 33 & Moderate \\
\hline Recurrence (distant) & OR 3.18 (1.56 to 6.46$)$ & 86 & High \\
\hline \multicolumn{4}{|l|}{ Overall analysis (survival) } \\
\hline Total follow-up (all studies) & HR 2.08 (1.71 to 2.52 ) & 71 & Moderate \\
\hline Total follow-up (stage I) & HR 4.20 (2.65 to 6.65$)$ & 55 & Moderate \\
\hline \multicolumn{4}{|c|}{ Subgroup analysis (overall recurrence) } \\
\hline Study size (>350 patients) & OR 4.40 (1.52 to 12.73$)$ & 88 & High \\
\hline Positive PLC \% (<5\%) & OR 10.01 (5.09 to 19.69$)$ & 11 & Low \\
\hline Study score (high quality) & OR 5.07 (1.70 to 15.19$)$ & 82 & High \\
\hline \multicolumn{4}{|l|}{ Subgroup analysis (survival) } \\
\hline Study score (high quality) & HR 1.74 (1.37 to 2.23 ) & 71 & Moderate \\
\hline
\end{tabular}

Please note, the degree of heterogeneity was graded as low $\left(1^{2}<25 \%\right)$, moderate $\left(I^{2}=25-75 \%\right)$ or high $\left(I^{2}>75 \%\right)$.

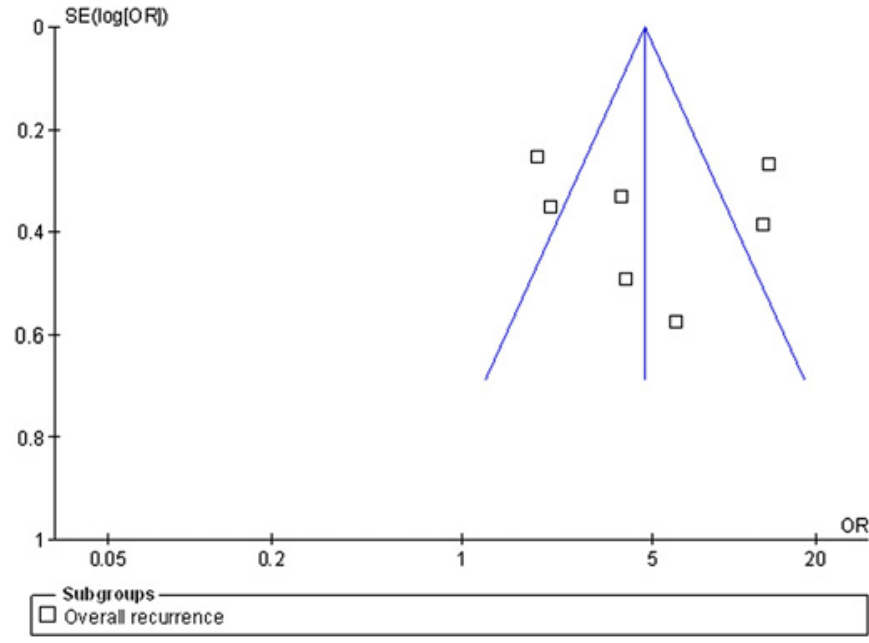

Figure 4 Funnel plot of analysis comparing overall recurrence at maximum follow-up (all patients).

positive cytology in the studies analysed. This may bring about the possibility that a significant proportion of stage I cancers may have been 'understaged'. Such a scenario may arise secondary to the current thoracic surgery guidelines for staging at the time of resection, which clearly request complete mediastinal lymph node resection. Positive PLC in such patients can therefore improve the accuracy of staging by identifying patients with residual microscopic disease which may not otherwise be detectable (prior to resection) and not accounted for using the TNM classification. In fact, the meta-analysis performed by Lim et al concluded that the effect of positive PLC would be to upstage a patient by one T category (up to a maximum of T4). ${ }^{13}$ This 'upstaging' would ultimately lead to a migration of patients from stage I to stage II, and a possible addition of postoperative chemotherapy to their treatment.

\section{Strengths and limitations}

This study is the first systematic review and quantitative analysis of the evidence on the prognostic effect of pre-resection PLC on lung cancer recurrence. The validity of any meta-analytical review can potentially be compromised by heterogeneity, clinical and statistical. As we described earlier, our conclusions are statistically significant. However, we recognise the moderate to high levels of heterogeneity within our results. Variation in inclusion criteria, patient characteristics, treatment protocols and operative technique explains the clinical heterogeneity. In our case, it was the variation in patient monitoring and reporting which most likely introduced the biggest error. Most of the studies did not report the monitoring methods used for recurrence and none specified the follow-up practice. Significant intraoperative factors exist which are known to potentially influence recurrence outcome, such as differing surgical practice and variation in the incidence and management of postoperative complications. Furthermore, a difference in the cytological techniques applied for detection of cancer cells in pleural lavage fluid, and the experience of the pathologist may have had a pronounced effect.

To investigate the extent to which statistical heterogeneity might have affected our conclusions, we performed a subgroup analysis. Interestingly, for all four subgroups (yearly follow-up, study size, positive PLC $<5 \%$ and quality assessment), OR and HR were $>1$ and statistically significant. Therefore, our conclusion that positive pre-resection PLC is a strong prognostic factor for recurrence is supported by a separate subgroup analysis. 
This study can be strengthened in several ways, some of which depend on an increased level of intra-study reporting. First, although the evidence seems to demonstrate a prognostic element to positive pre-resection PLC, this finding could have been further consolidated if recurrence data had been reported separately for stage I and II disease with stages III and IV excluded. Patients diagnosed with stage III and IV disease are routinely treated for systemic disease anyway. Second, separate recurrence data for each type of non-small cell lung cancer would be welcome and would add to the conclusions. Finally, we believe that authors should report in detail all investigations used for staging. Because not all institutions have a homogenous preoperative protocol, relevant funding and/or access to advanced techniques such as PET scans, it would have been desirable to compare studies in which pre-resection PLC was combined with these techniques and those studies in which it was not. Such a comparison would allow us to perceive whether the effect of pre-resection PLC on the staging process was significant, even when applied simultaneously with advanced investigatory techniques.

\section{Implications of our study and conclusions}

This article highlights the prognostic value of pre-resection detection of lung cancer cells in pleural wash-outs, for predicting postoperative recurrence outcomes in patients with lung cancer. It has also confirmed the findings of the two previous metaanalyses by Lim et al and Li et al which highlighted a link between unfavourable survival outcomes and positive preresection PLC. As positive pre-resection PLC is associated with higher overall, distant and local recurrence (and lower survival rates), cytological examination of pleural lavage fluid prior to curative lung cancer surgery may prove beneficial during the initial tumour staging process and thus subsequent management decisions.

Pre-resection PLC may be a standard diagnostic tool performed together with other invasive staging investigations (mediastinoscopy or mediastinotomy) before the planned operation. The techniques used will depend on the availability of trained personnel and the thoracic unit characteristics. A thoracoscopic procedure could be performed in university/teaching hospitals whereas a simple chest drain can be applied in units where minimally invasive procedures are not yet routine practice. Finally, PLC is performed prior to the resection, thus avoiding the issue of contamination encountered when performing post-resection PLC. This allows for a more definitive inference.

However, we appreciate that this meta-analysis is 'hypothesis generating' rather than conclusive and we believe that further research is required. In particular, our results suggest the need for a large-scale, multi-centre, prospective randomised trial of 'surgery' versus 'surgery and adjuvant chemotherapy' in patients with stage I disease found to have positive pre-resection PLC (with any type of recurrence as a primary endpoint). This will allow for a more scientifically rigorous approach to treatment of lung cancer.

Competing interests None.

Provenance and peer review Not commissioned; externally peer reviewed.

\section{REFERENCES}

1. American Cancer Society. Cancer Facts and Figures 2009. American Cancer Society. http://ww2.cancer.org/downloads/STT/500809web.pdf (accessed 9 Feb 2011).

2. Ries L, Eisner M, Kosary C. Cancer Statistics Review, 1975-2002. Bethesda, USA: National Cancer Institute, 2005.
3. Satoh Y, Hoshi R, Ishikawa $Y$, et al. Recurrence patterns in patients with early stage non-small cell lung cancers undergoing positive pleural lavage cytology. Ann Thorac Surg 2007:83:197-202.

4. Jiao X, Krasna MJ. Clinical significance of micrometastasis in lung and esophageal cancer: a new paradigm in thoracic oncology. Ann Thorac Surg 2002;74:278-84.

5. Mountain CF. Revisions in the international system for staging lung cancer. Chest 1997:111:1710-17.

6. Li YN, Shi HZ, Liang $\mathrm{OL}$, et al. Prognostic significance of pleural lavage cytology in patients with lung cancer: a meta-analysis. Lung Cancer 2008:60:183-92.

7. Spjut HJ, Hendrix VJ, Ramirez GA, et al. Carcinoma cells in pleural cavity washings. Cancer 1958;11:1222-5.

8. Aokage K, Yoshida J, Ishii G, et al. The impact on survival of positive intraoperative pleural lavage cytology in patients with non-small-cell lung cancer. J Thorac Cardiovasc Surg 2010:139:1246-52, 1252.e1

9. Taniguchi $\mathbf{Y}$, Nakamura $\mathrm{H}$, Miwa $\mathrm{K}$, et al. Prognostic significance of pleural lavage cytology after thoracotomy and before closure of the chest in lung cancer. Interact Cardiovasc Thorac Surg 2009;9:102-6.

10. Shintani Y, Ohta M, Iwasaki T, et al. Intraoperative pleural lavage cytology after lung resection as an independent prognostic factor for staging lung cancer. J Thorac Cardiovasc Surg 2009;137:835-9.

11. Kawachi R, Nakazato $Y$, Masui $K$, et al. Clinical significance of pleural lavage cytology for non-small cell lung cancer: is surgical resection valid for patients with positive pleural lavage cytology? Interact Cardiovasc Thorac Surg 2009;9:265-8.

12. Higashiyama M, Oda K, Okami J, et al. Prognostic value of intraoperative pleural lavage cytology for lung cancer without carcinomatous pleuritis: importance in patients with early stage disease during long-term follow-up. Eur J Cardiothorac Surg 2009;35:337-42.

13. Lim $\mathbf{E}$, Clough $\mathrm{R}$, Goldstraw $\mathrm{P}$, et al. Impact of positive pleural lavage cytology on survival in patients having lung resection for non-small-cell lung cancer: an international individual patient data meta-analysis. J Thorac Cardiovasc Surg 2010;139:1441-6.

14. The Cochrane Library. Cochrane Handbook for Systematic Reviews of Interventions 4.2.5 [updated May 2005]. Chichester, UK: John Wiley \& Sons, Ltd, 2005.

15. Moher D, Liberati A, Tetzlaff J, et al. Preferred reporting items for systematic reviews and meta-analyses: the PRISMA statement. BMJ 2009;339:b2535.

16. Stroup DF, Berlin JA, Morton SC, et al. Meta-analysis of observational studies in epidemiology: a proposal for reporting. Meta-analysis of Observational Studies in Epidemiology (MOOSE) group. JAMA 2000;283:2008-12.

17. Tierney JF, Stewart LA, Ghersi D, et al. Practical methods for incorporating summary time-to-event data into meta-analysis. Trials 2007;8:16.

18. Mantel N, Haenszel W. Statistical aspects of the analysis of data from retrospective studies of disease. J Natl Cancer Inst 1959:22:719-48.

19. DerSimonian R, Laird N. Meta-analysis in clinical trials. Control Clin Trials 1986:7:177-88.

20. Egger M, Davey Smith G, Altman DG. Systematic Reviews in Healthcare: MetaAnalysis in Context. London: BMJ Books, 1995

21. Galbraith RF. A note on graphical presentation of estimated odds ratios from several clinical trials. Stat Med 1988:7:889-94.

22. Stamou SC, Jablonski KA, Pfister AJ, et al. Stroke after conventional versus minimally invasive coronary artery bypass. Ann Thorac Surg 2002;74:394-9.

23. England MR, Gordon G, Salem M, et al. Magnesium administration and dysrhythmias after cardiac surgery. A placebo-controlled, double-blind, randomized trial. JAMA 1992;268:2395-402.

24. Higashiyama M, Doi 0 , Kodama K, et al. Pleural lavage cytology immediately afte thoracotomy and before closure of the thoracic cavity for lung cancer without pleura effusion and dissemination: clinicopathologic and prognostic analysis. Ann Surg Oncol 1997:4:409-15.

25. Buhr J, Berghäuser KH, Gonner $\mathrm{S}$, et al. The prognostic significance of tumor cell detection in intraoperative pleural lavage and lung tissue cultures for patients with lung cancer. J Thorac Cardiovasc Surg 1997;113:683-90.

26. Kondo H, Asamura $\mathrm{H}$, Suemasu $\mathrm{K}$, et al. Prognostic significance of pleural lavage cytology immediately after thoracotomy in patients with lung cancer. J Thorac Cardiovasc Surg 1993;106:1092-7.

27. Nakagawa T, Okumura N, Kokado Y, et al. Clinical relevance of intraoperative pleura lavage cytology in non-small cell lung cancer. Ann Thorac Surg 2007;83:204-8.

28. Vicidomini G, Santini M, Fiorello A, et al. Intraoperative pleural lavage: is it a valid prognostic factor in lung cancer? Ann Thorac Surg 2005;79:254-7.

29. Tomita M, Shimizu T, Matsuzaki Y, et al. Prognostic significance of carcinoembryonic antigen level in pleural lavage fluid for patients with lung adenocarcinoma. Ann Thorac Surg 2005;80:276-81.

30. Lim E, Ali A, Theodorou P, et al. Intraoperative pleural lavage cytology is an independent prognostic indicator for staging non-small cell lung cancer. J Thorac Cardiovasc Surg 2004;127:1113-18.

31. Okada M, Sakamoto T, Nishio W, et al. Pleural lavage cytology in non-small cell lung cancer: lessons from 1000 consecutive resections. J Thorac Cardiovasc Surg 2003; 126:1911-15

32. Dresler CM, Fratelli C, Babb J. Prognostic value of positive pleural lavage in patients with lung cancer resection. Ann Thorac Surg 1999;67:1435-9.

33. Hillerdal G, Dernevik L, Almgren SO, et al. Prognostic value of malignant cells in pleural lavage at thoracotomy for bronchial carcinoma. Lung Cancer 1998:21:47-52.

34. Okumura $\mathbf{M}$, Ohshima $\mathrm{S}$, Kotake $\mathrm{Y}$, et al. Intraoperative pleural lavage cytology in lung cancer patients. Ann Thorac Surg 1991;51:599-604. 